
December 1938

\title{
PORTABLE GEIGER COUNTER UNIT
}

\author{
By Leon F. Curtiss
}

\section{ABSTRACT}

A readily portable unit with entirely self-contained power supply, for operation of a low-voltage Geiger-Müller tube counter is described. The Neher-Harper circuit is incorporated in an integrating circuit which yields one pulse for approximately 60 counter pulses. The output pulse operates a Cenco counter. The sensitivity is such that 1 microgram of radium can be measured at 1 meter or 1 milligram at about 30 meters. This unit can therefore be used for measuring the gamma-ray activity of feebly active samples and also to detect slight contamination due to spilled or broken radium preparations. It also makes a very sensitive device for locating lost radium. Since it is operated entirely from dry batteries mounted in the case it can be used anywhere. The total weight is 13 pounds.

CONTENTS

I. Introduction

II. Description of eircuit $\ldots$

III. Geiger-Müller tube

IV. Mounting for the unit_... 781

V. Sensitivity $\ldots 782$

\section{INTRODUCTION}

Situations frequently arise where it is desirable to measure feeble gamma-ray activity under conditions where alternating-current mains are not available for power supply for Geiger-Müller tube counters. Examples are measurement of radium ores in their original location, detection of lost radium, and in surveys of radium dial painting establishments to detect concealed deposits of luminous powder which have accumulated over long periods of operation and may become a hazard to the health of the workers. A simple, compact, and sensitive device of this kind is described below, which is operated entirely from dry batteries contained in the case of the instrument.

\section{DESCRIPTION OF CIRCUIT}

The wiring diagram of the control circuit for operating the counter is shown in figure 1. The Neher-Harper ${ }^{1}$ circuit is incorporated in the first stage to permit the use of a large counter to increase the sensitivity and decrease the time required for observations, which is secured by the high counting rate which this circuit permits. The counter pulses from the Neher-Harper circuit are amplified and leveled in amplitude in the second stage. The third stage is a rectifier 
which enables the condenser, $C_{3}$, to be charged by the rectified pulses. As soon as the voltage on this condenser reaches the flashing voltage of the neon lamp, $N$, shunted about it, the condenser is discharged, which impresses a voltage pulse on the grid of the last tube actuating the Cenco counter, $Z$. Thus the circuit serves to integrate the pulses and a wide range of ratios between the number of Geiger-Müller pulses and counts registered by the Cenco counter can be obtained by adjusting the constants of the input to the last stage.

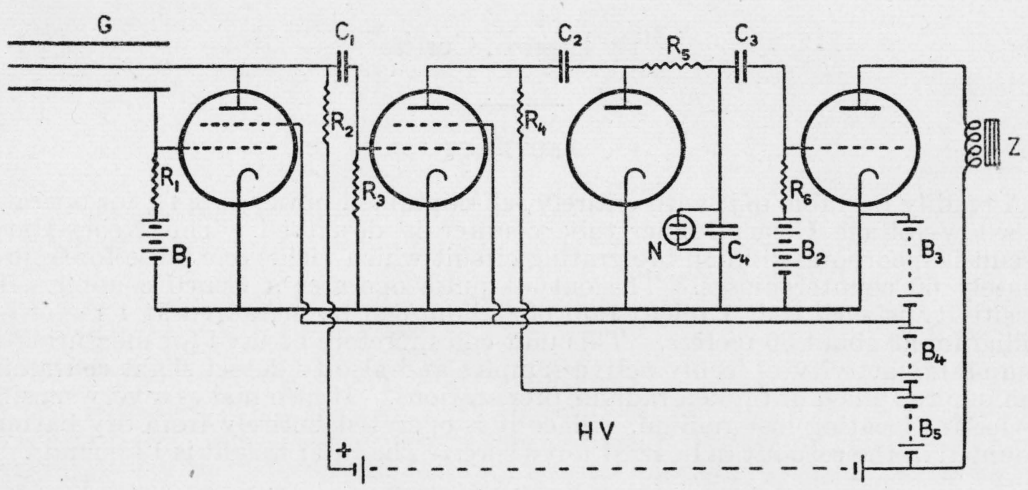

FIGURE 1.-Wiring diagram of integrating amplifier for Geiger-Müller counter.

$G$, Geiger-Müller tube; $Z$, Cenco counter; $N, T 41 / 2$ lamp.

Circuit constants are as follows:

$\begin{array}{lll}R_{1}, R_{5}=20 \text { meg. } & C_{1}, C_{2}=0.0001 \mathrm{mf} . & B_{1}=6 \mathrm{v} . \\ R_{2}=5 \text { meg. } & B_{2}=10 \text { to } 15 \mathrm{v} . \\ R_{3}=1 \text { meg. } & C_{3}, C_{4}=0.01 \mathrm{mf} & B_{3}=15 \text { to } 30 \mathrm{v} . \\ R_{3}=1 \text { meg. } & & B_{3}=90 \mathrm{v} . \\ R_{0}=10 \text { meg. } & & B_{3}=45 \mathrm{v} . \\ & & H=405 \text { to } 450 \mathrm{v} . \\ & & \text { (Eveready X-180) }\end{array}$

Since observations must be continued over a considerable time to secure reasonably accurate measurements, the natural variations of the flashing voltage of the neon lamp are averaged out quite adequately since at most these variations are of the order of 1 percent. Thus the neon lamp introduces no noticeable error into the final results, which from their nature under usual conditions do not exceed an accuracy of 2 or 3 percent.

\section{GEIGER-MÜLLER TUBE}

One obstacle to the satisfactory design of portable counter units with self-contained power supply in the past has been the high voltrge, usually about 1,200 volts, required for operation of the Geiger-Müller tube counter. Recent work in this laboratory has shown that it is possible to operate counters up to 5 or $6 \mathrm{~cm}$ in diameter, containing gas at 6 - or $7-\mathrm{cm} \mathrm{Hg}$ pressure, at voltages between 450 and 650 volts. This reduction in voltage has been obtained by using a mixture of argon and hydrogen in the tube. There seems to be a fairly critical ratio of hydrogen to argon at which the counting voltage is much lower than for either gas alone at the same pressure. This condition has been obtained by trial in the counters we have made so that we have no exact information as to the proper proportions of the two gases. 


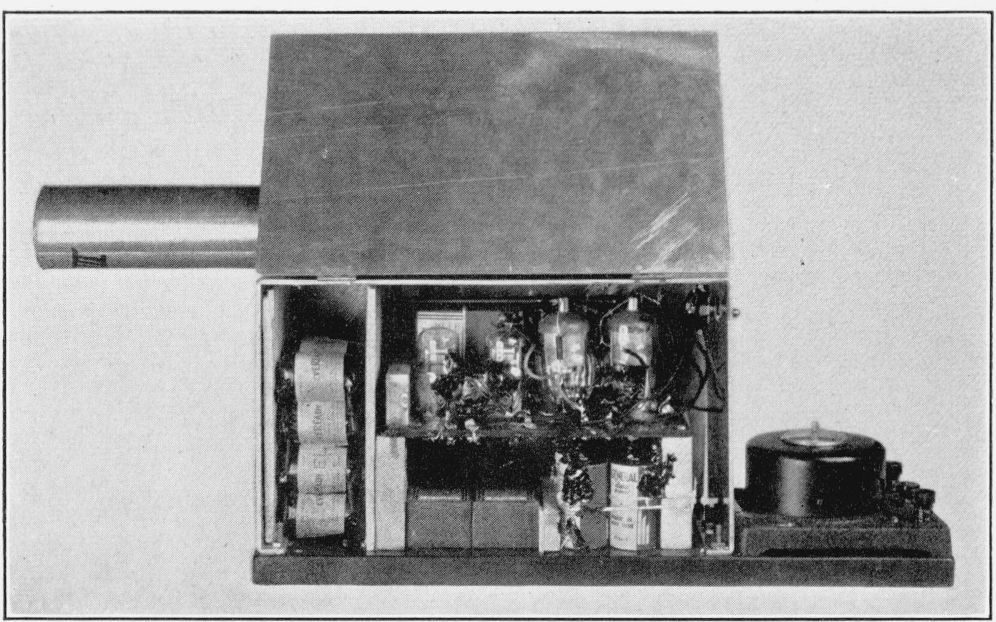

FIGURE 3.-Interior view of case, showing the location of batteries and integrating amplifier.

Cenco counter at right.

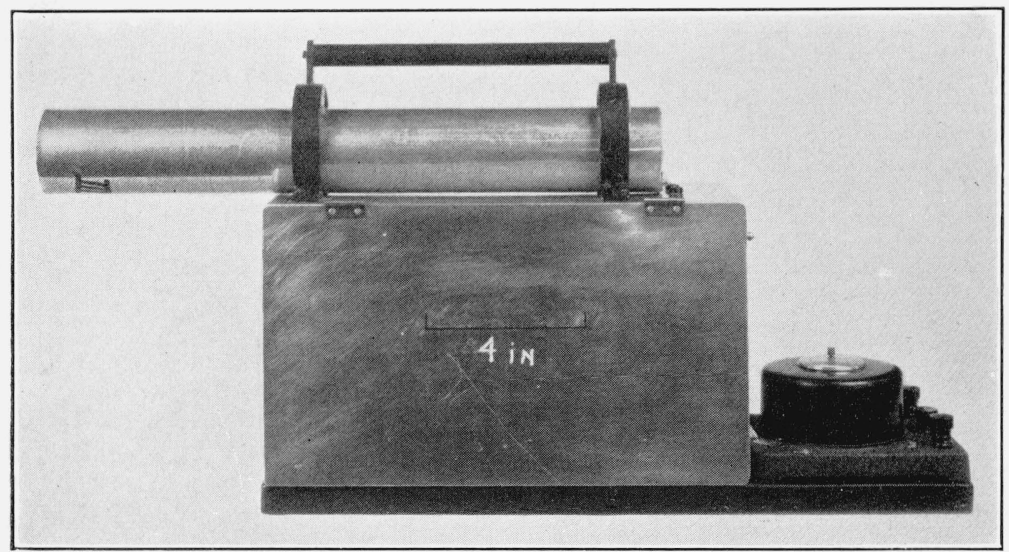

FIGURE 4.-External view of completely assembled instrument with Geiger-Müller tube mounted in lighttight cylinder on top of the case. 
Another recent development due to Duffenback, Lifschutz, and Slawsky ${ }^{2}$ has made possible the production of tube counters with exceptionally stable characteristics and remarkably flat "plateaus." Our procedure has varied somewhat from that recommended by these authors. We find it sufficient to pass a feeble alternating-current discharge between the wire and cylinder of individual counters using hydrogen which is frequently changed, as recommended by them.

An example of the operating characteristics of a counter prepared in this way and filled with a hydrogen-argon mixture at $7-\mathrm{cm} \mathrm{Hg}$ pressure is shown in figure 2 , which shows the variation of counting rate with voltage. The diameter of the outer cylinder, made from 100-mesh copper gauze, as recommended by Evans, ${ }^{3}$ was in this case $4.5 \mathrm{~cm}$. Tests made in the Bureau's laboratory have verified the increase in sensitivity over a smooth metallic cylinder, which Evans reports. Referring to the curve in figure 2 , it can be seen that the flat part of the curve from about 500 to 650 volts is very nearly horizontal. The counting rate in this range is therefore practically independent of voltage. This is equally a test of the linearity of the integrating circuit since pulses delivered to the integrating condenser must be sensibly equal to obtain a plateau of this type. ${ }^{4}$

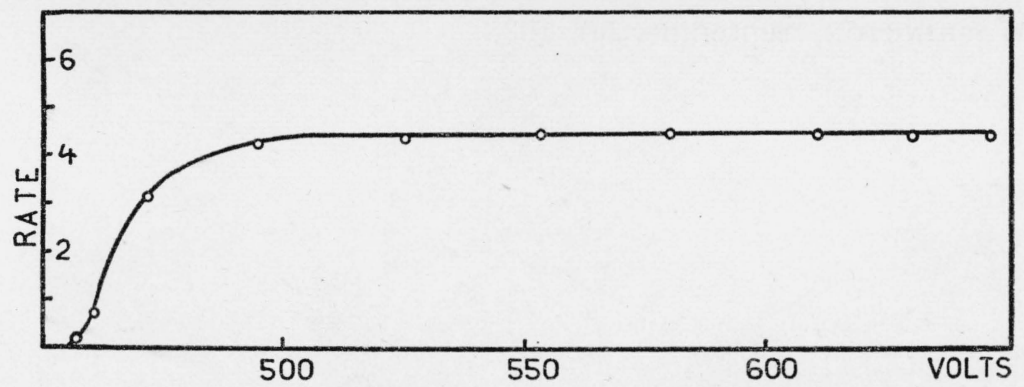

FIGURE 2.-Curve showing variation of counting rate with voltage for Geiger-Müller tube when uscd in the integrating circuit shown in figure 1.

Counters of this type, sealed in Pyrex, have been in use over a year without appreciable change in sensitivity or operating characteristics.

\section{MOUNTING FOR THE UNIT}

Recent developments in commercial dry batteries have provided 45-volt units of exceptionally light weight, in the neighborhood of 2 ounces. Consequently, it is quite possible to inclose the batteries indicated in figure 1 within the case for the integrator circuit. The mounting and inclosure used for one of our units is shown in figures 3 and 4 , which are photographs of the complete unit. Figure 3 shows the compartment inclosing the batteries and integrating circuit open, and figure 4 shows the compartment closed ready for use. The counter is mounted horizontally inside a tubular aluminum shield. The total weight is 13 pounds.

${ }^{2}$ Phys. Rev. 52, 1231 (1937).

3 Rev. Sci. Instr. 7, 444 (1936).

4 Attention must of course be given to the batteries, as they deteriorate with age. Instead of checking them by a voltmeter, which does not necessarily indicate their open circuit voltage it is better to test the instrument as a unit by a radioactive preparation of known magnitude. The National Bureau of Standards is prepared to issue feeble standards for this purpose. 


\section{SENSITIVITY}

The sensitivity of this unit, using a 100-mesh gauze counter 4.5 by $25 \mathrm{~cm}$, is such that 1 microgram of radium at 1 meter increases the rate of the Cenco counter by about 12 counts per hour. Thus this instrument can readily be used to detect radium ores and stray deposits of luminous powder in radium dial painting plants. It also may serve as a very sensitive detector for lost radium preparations of the type used for cancer treatment. An unshielded preparation of 1 milligram of radium can be detected at 25 to 30 meters. Therefore, the presence of lost radium can be determined readily, even when lodged in inaccessible locations. The complete portability of the instrument makes its use convenient and enables explorations over large areas to be made with ease.

The author acknowledges the help of A. V. Astin in connection with the details of the integrating circuit, and the assistance of $\mathrm{B} . \mathrm{W}$. Brown and L. L. Stockmann in constructing and testing the complete unit.

Washington, September 30, 1938. 